\begin{tabular}{|c|c|}
\hline Title & Numerical Modeling of Photonic Crystal Fibers \\
\hline Author(s) & Saitoh, Kunimasa; Koshiba, Masanori \\
\hline Citation & $\begin{array}{l}\text { JOURNAL OF LIGHTWA VE TECHNOLOGY, 23(11), 3580-3590 } \\
\text { https://doi.org/10.1109/JLT .2005.855855 }\end{array}$ \\
\hline Issue Date & 2005-11 \\
\hline Doc URL & http:/hdl.handle.net/2115/1404 \\
\hline Rights & $\begin{array}{l}\text { @ 2005 IEEE. Personal use of this material is permitted. However, permission to reprint/republish this material for } \\
\text { advertising or promotional purposes or for creating new collective works for resale or redistribution to servers or lists, } \\
\text { or to reuse any copyrighted component of this work in other works must be obtained from the IEEE }\end{array}$ \\
\hline Type & article \\
\hline File Information & JLT23-11.pdf \\
\hline
\end{tabular}

Instructions for use 


\title{
Numerical Modeling of Photonic Crystal Fibers
}

\author{
Kunimasa Saitoh, Member, IEEE, Member, OSA, and Masanori Koshiba, Fellow, IEEE, Fellow, OSA
}

Invited Paper

\begin{abstract}
Recent progress on numerical modeling methods for photonic crystal fibers (PCFs) such as the effective index approach, basis-function expansion approach, and numerical approach is described. An index-guiding PCF with an array of air holes surrounding the silica core region has special characteristics compared with conventional single-mode fibers (SMFs). Using a full modal vector model, the fundamental characteristics of PCFs such as cutoff wavelength, confinement loss, modal birefringence, and chromatic dispersion are numerically investigated.
\end{abstract}

Index Terms-Finite-element method, holey fiber, microstructured optical fiber, numerical modeling, photonic band-gap fiber, photonic crystal fiber.

\section{INTRODUCTION}

$\mathbf{P}$ HOTONIC crystal fibers (PCFs) [1]-[3], also called holey fibers or microstructured optical fibers, have been under intensive study for the past several years as they offer a number of unique and useful properties not achievable in standard silica glass fibers. PCFs fall into two basic categories. The first one, an index-guiding PCF [4], [5], is usually formed by a central solid defect region surrounded by multiple air holes in a regular triangular lattice and confines light by total internal reflection like standard fibers. The second one uses a perfect periodic structure exhibiting a photonic band-gap (PBG) effect at the operating wavelength to guide light in a low index core region, which is also called PBG fiber (PBGF) [6], [7].

Numerical simulations play an important role for the design and modeling of PCFs. So far, various modeling methods in which not only a full-vector model but also an approximatescalar model is used have been developed such as effective index approach [5], [8], plane-wave expansion (PWE) method [9]-[12], localized-function method [13]-[16], multipole method (MM) [17]-[21], beam propagation method (BPM) [22]-[24], finite-difference method (FDM) [25], finitedifference time-domain method (FDTD) [26]-[28], boundary element method (BEM) [29], [30], and finite-element method (FEM) [31]-[54]. An approximate-scalar model is a valuable tool for aiding fabrication efforts because it is easy to use and provides good qualitative information. However, in order to model PCFs accurately, it is crucial to use a full-vector model. In particular, a complete vector model is necessary for predicting sensitive quantities such as dispersion and birefringence.

Manuscript received November 15, 2004; revised June 6, 2005.

The authors are with the Division of Media and Network Technologies, Hokkaido University, Sapporo 060-0814, Japan (e-mail: ksaitoh@ist. hokudai.ac.jp).

Digital Object Identifier 10.1109/JLT.2005.855855

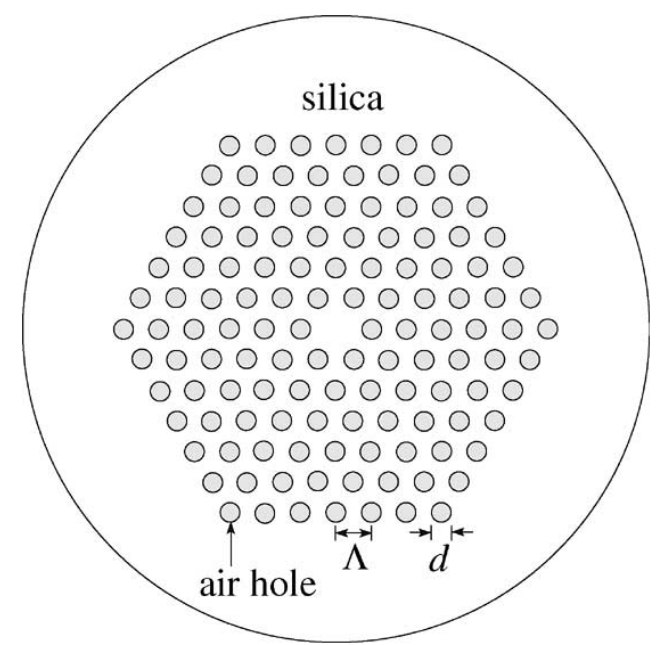

Fig. 1. Schematic representation of a PCF.

The choice of modeling tool can impact the computational time, required computational resources, and limitations of the methods, so it is important to explore the usefulness and limitations of each method.

In this paper, first, recent progress on numerical modeling methods of PCFs such as the effective index approach, basisfunction expansion approach, and numerical approach is described. Next, using a full-vector mode solver based on the FEM with curvilinear hybrid edge/nodal elements [55] that can avoid spurious solutions and can accurately model curved boundaries of air holes in PCFs, fundamental characteristics of index-guiding PCFs such as cutoff wavelength, confinement loss, modal birefringence, and chromatic dispersion are numerically investigated.

\section{AnAlysis Method}

\section{A. Effective Index Approach}

The cross section of a typical index-guiding PCF with sixfold rotational symmetry is schematically shown in Fig. 1, where $\Lambda$ is the hole pitch (center-to-center distance between the holes) and $d$ is the hole diameter.

The first approach developed for PCFs was the effective index approach [5], [8] based on a very simple scalar model using an effective cladding index. In this model, first, an effective index for the periodically repeated hole-in-silica structure is evaluated and then the microstructured cladding region is replaced by a uniform medium with a properly chosen effective 
index, resulting in an equivalent step index fiber (SIF) consisting of a core and a cladding region. Using this simple model and the well-established fiber theory, we can obtain a qualitative information about PCFs with perfect hexagonal symmetry. The core is pure silica, but the effective cladding index is determined by using the propagation constant of the lowest-order fundamental mode propagating in the periodically repeated hole-in-silica structure without any defects. The propagating modes in such an infinite cladding material are called spacefilling modes, the propagation constants of which are strongly dependent on the operating wavelength $\lambda$.

The propagation constants of the space-filling modes are usually calculated by solving an approximate scalar-wave equation within a unit cell centered on one of the air holes. By reflection symmetry, the Neumann condition is enforced on each cell edge, namely, the normal derivative of the cladding mode field to the edge must be zero. In general, however, the hexagonal cell shape is approximated by a circular one with radius of $\Lambda / 2$ because of the difficulty in solving the boundary-value problem with hexagonal boundaries. The propagation constant of the resulting fundamental space-filling mode (FSM) $\beta_{\mathrm{FSM}}$ is used to define the effective cladding index as $n_{\mathrm{FSM}}=\beta_{\mathrm{FSM}} / k_{0}$, with $k_{0}$ being the free-space wavenumber.

Recently, an analytical vector solution for the effective cladding index has been reported [56]. Also in this solution, the hexagonal unit cell is replaced by the corresponding circular one. It is pointed out [56] that choices of the radius of the circle influence the accuracy in $n_{\mathrm{FSM}}$ and that the choice of $\Lambda / 2$ yields results in good agreement with those of the full-vector PWE method [9]. Discrepancies in the values of $n_{\mathrm{FSM}}$ between scalar and vector approaches are also demonstrated [56].

\section{B. Basis-Function Expansion Approach}

Although the effective index approach can provide good qualitative information about PCFs, this approach is unable to accurately predict modal properties such as dispersion or birefringence. These quantities depend critically on PCF geometries. An early full-vector model for PCFs has been based on a modal decomposition technique using various basis functions such as sinusoidal functions [9], [10], Hermite-Gaussian functions [15], [16], and cylindrical functions [17], [18].

One of the most widely used is the PWE method [9], [10]. As the name implied, this method is based on the PWE of the electromagnetic field (in PWE, as usual, three components of the magnetic field) using Bloch's theorem. Also, the positiondependent permittivity (dielectric constant) is expressed as a Fourier series expansion. In order to treat a spatial defect such as a core region, a superperiodicity including the defect is introduced where the defect is also periodically repeated. This supercell approximation can accurately determine the properties of the defect region if the supercell is large enough to ensure that neighboring defects are uncoupled. The modal fields and their propagation constants may be found by solving a matrix eigenvalue problem that is derived from a variational method based on minimization of the functional corresponding to the wave equation. The PWE has been effectively applied not only to index-guiding PCFs [9], [10] but also to PBGFs [11], [12].
PWE involves defining the supercell over a restricted region and using periodic boundary conditions to extend the structure spatially, and therefore, the applicability to PCFs that do not need to be periodic is somewhat restricted. An alternative approach is the localized-function method (LFM) [15], [16]. As the guided modes in PCFs are localized in the defect core region, their modal fields are well described using localized Hermite-Gaussian functions. The LFM takes advantage of mode localization, and thus, a modest number of functions are required to accurately model the guided modes, resulting in less computational efforts, compared with the PWE. By decomposing the electromagnetic field (in LFM, as usual, three components of the electric field) and the index defect into localized Hermite-Gaussian functions, and the lattice of air holes using sinusoidal functions, each quantity can be represented efficiently and accurately without requiring the use of too many expansion terms [16]. Again, the wave equation is reduced to a matrix eigenvalue problem that is solved for the modal fields and their propagation constants. The LFM based on an approximate-scalar model is also available [13], [14].

If all the air holes are assumed to be circular, the modal fields can be expanded in cylindrical harmonic functions. This approach exploiting the local circular geometry is called the MM [17]-[21], which can describe the leaky nature of PCFs with a finite number of air holes. In the proximity of each air hole, the electromagnetic field (in MM, as usual, two longitudinal axial components of the electromagnetic field) is expressed in terms of Bessel and Hankel functions using local cylindrical coordinates. Inside each air hole, on the other hand, the axial electric and magnetic fields are expressed in terms of regular Bessel functions only. In order to obtain relations between all the expansion coefficients, boundary conditions on hole surfaces are used.

\section{Numerical Approach}

Although the basis-function expansion approach can accurately predict the modal properties such as dispersion and birefringence, it is difficult to apply it to more complicated fibers with noncircular air holes and/or longitudinally varying structures. Recently, published models utilize other direct numerical analysis techniques such as BPM [22]-[24], FDM [25], FDTD [26]-[28], BEM [29], [30], and FEM [31]-[54].

In the FEM, instead of solving the wave equation, the corresponding functional to which a variational method is applied is set up, where the fiber cross section is divided into the so-called elements, an equivalent discretized model for each element is constructed, and then all the element contributions to the whole fiber cross section are assembled, resulting in a matrix eigenvalue problem with nodal variables as unknowns, in contrast to the basis-function expansion approach taking expansion coefficients as unknowns. As a result, the matrices derived from the FEM and the basis-function expansion approach become sparse and dense, respectively.

For the characterization of longitudinally varying PCFs, the BPM is clearly the natural choice. Even the approximate-scalar BPM based on the fast Fourier transform (FFT-BPM) is able to describe some features of PCFs [22], [23]. This standard 


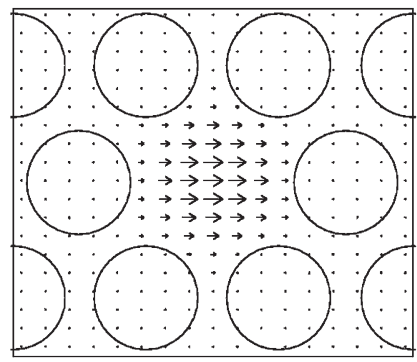

(a)

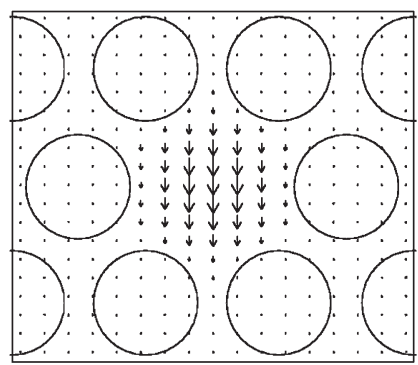

(b)

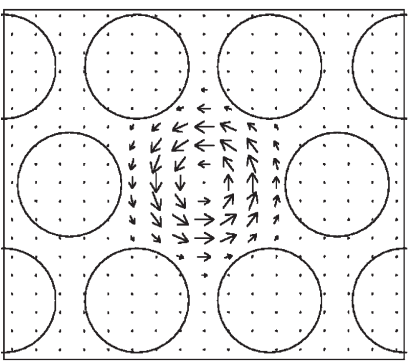

(a)

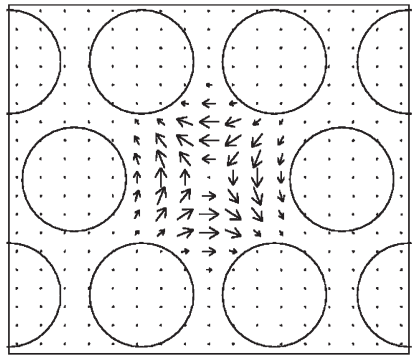

(c)

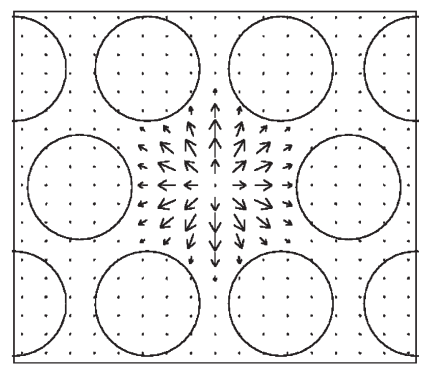

(b)

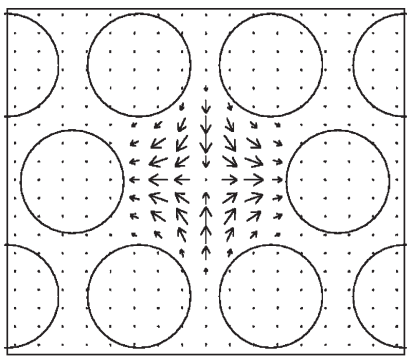

(d) structure is assumed, so the polarization effect cannot be taken into account. Recently, the full-vector BPM based on the FEM (FE-BPM) [35] or the FDM (FD-BPM) [24] has been applied to compute the propagation constants and the modal profiles in high-contrast PCFs. The FE-BPM has been effectively applied to investigating the problem of leakage due to finite number of arrays of air holes in PCFs [35].

Although the BPM is currently the most widely used for the study of light propagation in longitudinally varying optical waveguides, it is difficult to take into account backward reflecting waves. One method used to study distributed reflection and diffraction at arbitrary angle is the FDTD technique. This technique is very powerful and versatile and has been introduced and adapted to PCFs [26]-[28]. Splice losses between a standard SIF and a PCF, and spot-size and numerical aperture conversion phenomena in a tapered PCF are numerically investigated [26], [27].

\section{GUIDED MODES IN PCFS}

In an index-guiding PCF, the core index is greater than the average index of the cladding because of the presence of air holes, and the fiber can guide the light by total internal reflection as a standard fiber does. That is, the guided light has an effective index $n_{\text {eff }}$ that satisfies the condition

$$
n_{\mathrm{co}}>n_{\mathrm{eff}}=\frac{\beta}{k_{0}}>n_{\mathrm{FSM}}
$$

where $\beta$ is the propagation constant along the fiber axis, $n_{\mathrm{co}}$ is the core index, and $n_{\mathrm{FSM}}$ is the cladding effective index of the FSM. In the case of a PCF made from pure silica, $n_{\text {co }}$ is reduced to the index of silica.

Fig. 2 shows the transverse electric field vector distributions of the fundamental modes in a multimode PCF, where $\Lambda=2.3 \mu \mathrm{m}, d=1.8 \mu \mathrm{m}$, the background index of silica is assumed to be 1.45 , and the operating wavelength is $0.46 \mu \mathrm{m}$. The fundamental modes in Fig. 2 resemble the linearly polarized modes $\mathrm{LP}_{01}$ in the conventional SIFs. As in the SIFs, the linearly polarized fundamental modes $\mathrm{HE}_{11}^{x}$ and $\mathrm{HE}_{11}^{y}$ are

Fig. 3. Transverse electric field vector distributions of (a) $\mathrm{TE}_{01}$, (b) $\mathrm{TM}_{01}$, (c) $\mathrm{HE}_{21}^{\prime}$, and (d) $\mathrm{HE}_{21}^{\prime \prime}$ modes.

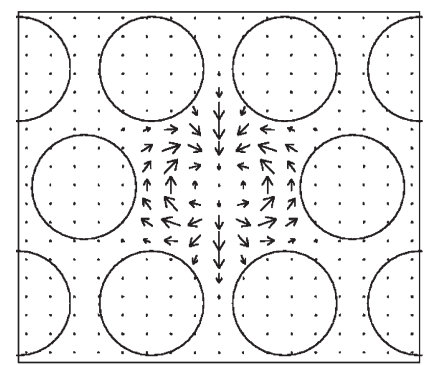

(a)

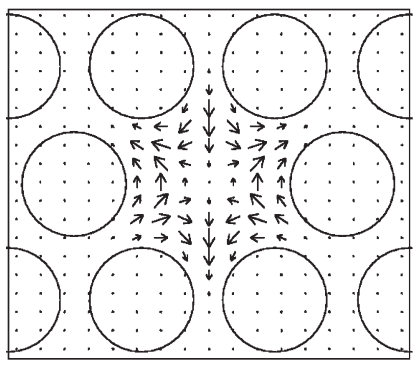

(c)

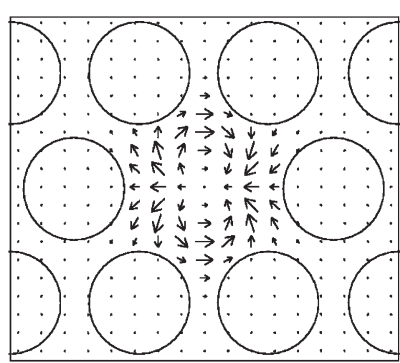

(b)

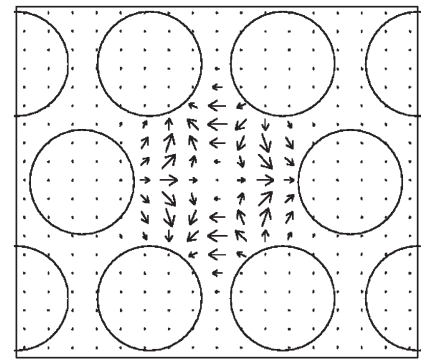

(d)
Fig. 4. Transverse electric field vector distributions of (a) $\mathrm{EH}_{11}^{\prime}$, (b) $\mathrm{EH}_{11}^{\prime \prime}$, (c) $\mathrm{HE}_{31}^{\prime}$, and (d) $\mathrm{HE}_{31}^{\prime \prime}$ modes.

degenerate. The degeneracy in these two fundamental $\mathrm{HE}_{11}$ states has already been confirmed by using the full-vector MM [17], the full-vector FEM [32], and the full-vector BPM [24]. Figs. 3-5 show the higher-order modes. By analogy with the SIFs, we call these guided modes TE, TM, HE, and EH. The higher-order modes in Figs. 3-5 look like the $\mathrm{LP}_{11}, \mathrm{LP}_{21}$, and $\mathrm{LP}_{02}$ modes, respectively.

The cutoff wavelength of each higher-order mode is determined by using the effective cladding index $n_{\mathrm{FSM}}$. The cutoff of the higher-order mode occurs when its effective refractive index becomes equal to the value of $n_{\mathrm{FSM}}$. The accurate 


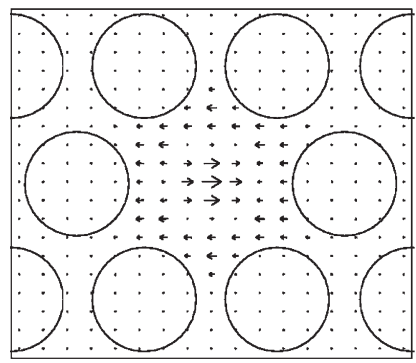

(a)

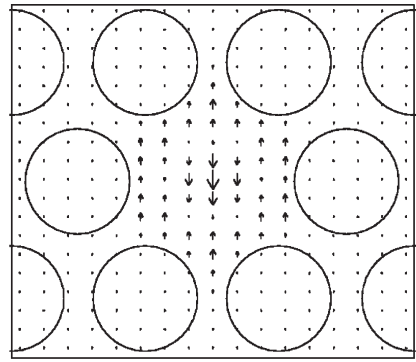

(b)
Fig. 5. Transverse electric field vector distributions of (a) $\mathrm{HE}_{12}^{x}$ and (b) $\mathrm{HE}_{12}^{y}$ modes.

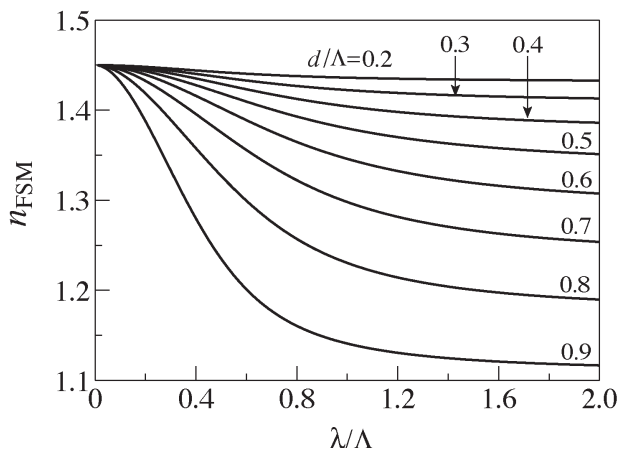

Fig. 6. Effective index of the FSM for the PCF with different hole diameters.

value of $n_{\mathrm{FSM}}$ can be determined by applying the full-vector FEM to the so-called elementary piece in the cladding region, which acts as a boundary-less propagation medium [31], [34]. Fig. 6 shows a wavelength dependence of the effective index of the FSM $n_{\mathrm{FSM}}$ with different hole diameters, where the background index is assumed to be 1.45 . We can see the strong wavelength dependence of $n_{\mathrm{FSM}}$ that facilitates the unusual chromatic dispersion properties of PCFs.

\section{NORMALIZED FREQUENCY OF PCFS}

The $V$ parameter (normalized frequency) is frequently used in the design of conventional SIFs and is given by

$$
V=\frac{2 \pi}{\lambda} a \sqrt{n_{\mathrm{co}}^{2}-n_{\mathrm{cl}}^{2}}
$$

that must be less than 2.405 for the fiber to be single mode, where $\lambda$ is the operating wavelength, $a$ is the core radius, $n_{\mathrm{co}}$ is the core index, and $n_{\mathrm{cl}}$ is the cladding index. Recently, it has been reported that the fundamental properties of index-guiding PCFs such as cutoff wavelength, mode field diameter (MFD), splice loss, and so on, can be easily estimated without the need for heavy numerical computations by appropriately defining the $V$ parameter [57]. By analogy with SIFs, the effective $V$ parameter $V_{\text {eff }}$ for PCFs can be defined as

$$
V_{\mathrm{eff}}=\frac{2 \pi}{\lambda} a_{\mathrm{eff}} \sqrt{n_{\mathrm{co}}^{2}-n_{\mathrm{FSM}}^{2}}
$$

where $a_{\text {eff }}$ is the effective core radius. To adapt the concept of $V$ parameter to PCFs, the value of $a_{\text {eff }}$ should be determined. We have confirmed from the full-vector FEM analysis that

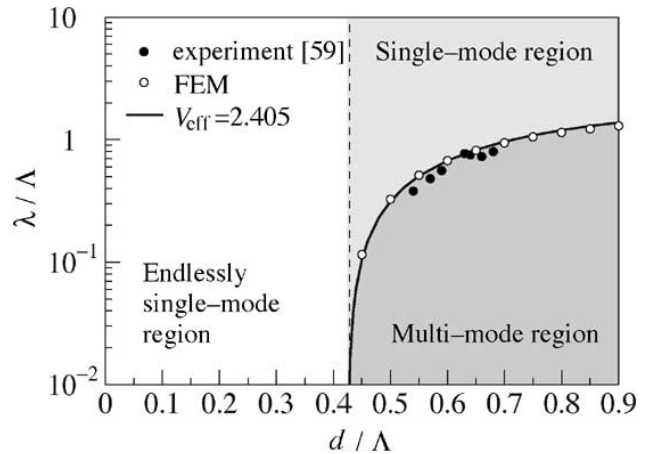

Fig. 7. Relative cutoff wavelength $\lambda / \Lambda$ as a function of relative hole diameter $d / \Lambda$.

for small hole diameters, $d / \Lambda<0.43$, the PCFs are endlessly single mode. Until now, various effective core radii have been proposed, such as $a_{\mathrm{eff}}=\Lambda, \Lambda / 2$, and $\Lambda-d / 2$. When using these effective core radii, for the PCFs with hole diameter $d / \Lambda<0.43$, the effective $V$ parameter $V_{\text {eff }}$ is not less than 2.405 at all wavelengths. So, to use the definition of the cutoff $V$ value as in conventional SIFs $(V=2.405)$, we have to choose the value of $a_{\text {eff }}$ to be $\Lambda / \sqrt{3}$ [34], [57]. Mortensen et al. proposed another effective $V$ parameter for PCF [58]. However, this definition is intrinsically different from the original $V$ parameter definition in SIF theory and corresponds to the normalized transverse attenuation constant $W$ parameter. So we adopt the $V$ parameter definition of (3). From (3) with $a_{\text {eff }}=\Lambda / \sqrt{3}$, the cutoff condition is given by $V_{\text {eff }}=2.405$, as in conventional SIFs, and therefore the $V$ parameter in (3) enables us to apply the design principle for standard SIFs straightforwardly to PCFs [57].

Fig. 7 shows the relative cutoff wavelength $\lambda / \Lambda$ as a function of relative hole diameter $d / \Lambda$ obtained from the cutoff condition $V_{\text {eff }}=2.405$. The calculated cutoff wavelengths obtained from (3) and $V_{\text {eff }}=2.405$ agree well with the experimental results [59] and the results of direct numerical simulation based on the FEM. We can see that PCFs with relative hole diameters less than 0.43 can be endlessly single moded and that even for large air holes single-mode operation is possible for wavelengths above a cutoff wavelength. If the value of $d / \Lambda$ is given, a given cutoff wavelength can be obtained by scaling the fiber structure. This allows for the development of singlemode fibers (SMFs) with very small or large MFDs, which are useful for highly nonlinear SMF application, high bit rate wideband data transmission, single-mode high power delivery, and so on.

Next, to evaluate the MFD, we introduce the Marcuse formula [60] as

$$
\frac{w}{a_{\mathrm{eff}}}=0.65+\frac{1.619}{V_{\mathrm{eff}}^{\frac{3}{2}}}+\frac{2.879}{V_{\mathrm{eff}}^{6}}
$$

where $w$ is the half MFD and is called the effective modal spot size. In Fig. 8, calculated MFD results are shown as a function of wavelength for different hole sizes. The experimental results for two PCFs fabricated by Crystal Fiber A/S [61], namely, LMA-5 $(\Lambda=2.9 \mu \mathrm{m}, d / \Lambda=0.44)$ and LMA-8 $(\Lambda=5.6 \mu \mathrm{m}$, $d / \Lambda=0.49)$, are also shown. The results of (3) and (4) are in 


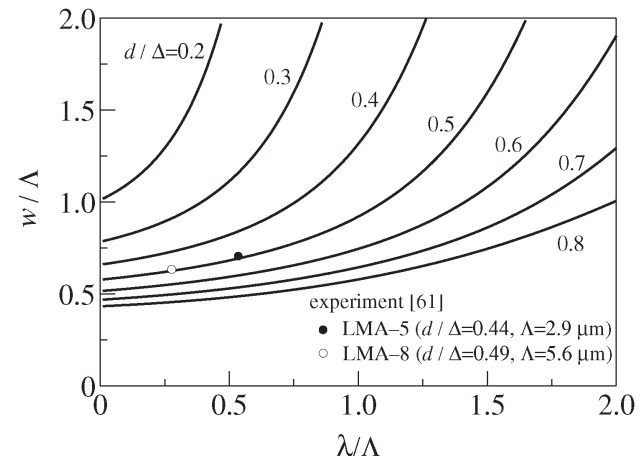

Fig. 8. Mode field diameter of PCFs as a function of wavelength for different hole sizes.

good agreement with measured data [61]. Knowledge of MFD is an important starting point in the context of nonlinearities. Due to the high index contrast between silica and air, the PCF technology can offer a smaller MFD compared to standard fiber technology, which is useful for broadband supercontinuum generation, soliton pulse transmission, nonlinear optical loop mirror, optical signal processing, and so on. In addition, PCFs have a large potential for single-mode large-mode-area optical fibers with low nonlinearity because of their endlessly singlemode properties combined with large MFDs.

With the modal spot size, Gaussian beam propagation theory states that the splice loss $L_{s}$ between a PCF and a SMF is given by [60]

$$
L_{s}=-10 \log _{10}\left\{\left(\frac{2 w w_{\mathrm{SMF}}}{w^{2}+w_{\mathrm{SMF}}^{2}}\right)^{2} \exp \left(\frac{-2 \delta^{2}}{w^{2}+w_{\mathrm{SMF}}^{2}}\right)\right\}
$$

where $2 w_{\mathrm{SMF}}$ is the MFD of the SMF that is also calculated with (4) and $\delta$ is a transverse offset. In evaluating the splice losses, we assume that the SMF is an SM28 fiber. Fig. 9(a) and (b) shows the splice losses between the PCF and the SM28 fiber as a function of hole pitch $\Lambda$ for $d / \Lambda$ from 0.25 to 0.55 with $\delta=0$ and as a function of offset $\delta$, respectively, where an operating wavelength is $1.55 \mu \mathrm{m}$. For comparison, the measured results [62] and the numerical results obtained by use of the FEM [62] are also plotted. The results based on (5) agree approximately with the measured and numerical results.

\section{Confinement Loss And EFFective MODE AREA IN PCFS}

The losses in PCFs occur for a number of reasons, such as intrinsic material absorption loss, structural imperfection loss, Rayleigh scattering loss, confinement loss, and so on. Fabrication-related losses can be reduced by carefully optimizing the fabrication process [63]. Confinement loss is an additional form of loss that occurs in single-material fibers. PCFs are usually made from pure silica, and so the guided modes are inherently leaky [18] because the core index is the same as the index of the outer cladding without air holes. This confinement loss can be reduced exponentially by increasing the number of rings of air holes that surround the solid core, and

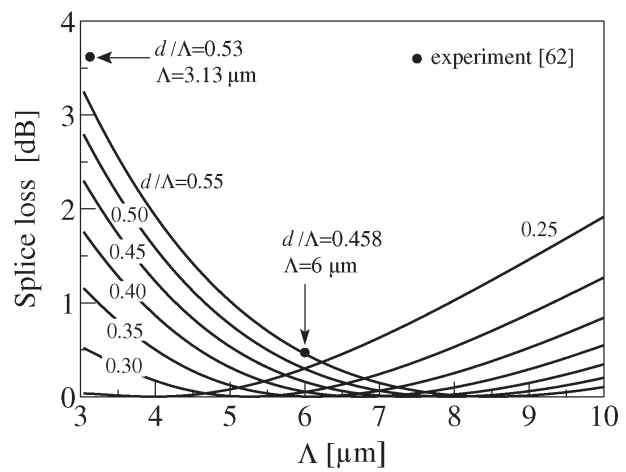

(a)

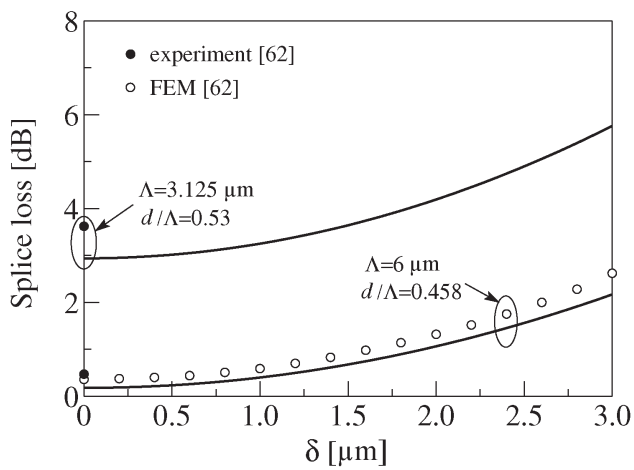

(b)

Fig. 9. Splice loss between the PCF and the SMF (a) as a function of hole pitch $\Lambda$ for $d / \Lambda$ from 0.25 to 0.55 with $\delta=0$ and (b) as a function of offset $\delta$, where an operating wavelength is $1.55 \mu \mathrm{m}$.

is determined by the geometry of the structure. It is important to know how many numbers of rings of air holes are required to reduce the confinement loss under the Rayleigh scattering limit for practical fabrication process.

Fig. 10 shows the normalized confinement loss of the fundamental mode in PCFs with finite cross sections as a function of the normalized effective area, taking the ratio of hole diameter to pitch ratio $d / \Lambda$ as a parameter, where $N$ is the number of rings of air holes and the background silica index is assumed to be 1.45 . The effective area of the fiber core $A_{\text {eff }}$ is defined as [64]

$$
A_{\mathrm{eff}}=\frac{\left(\iint_{S}\left|\boldsymbol{E}_{t}\right|^{2} \mathrm{~d} x \mathrm{~d} y\right)^{2}}{\iint_{S}\left|\boldsymbol{E}_{t}\right|^{4} \mathrm{~d} x \mathrm{~d} y}
$$

where $\boldsymbol{E}_{t}$ is the transverse electric field vector and $S$ denotes the whole fiber cross section. As expected, increasing the airhole size, the mode becomes more confined, and thus the effective area and the confinement loss are both reduced. Also, increasing the number of rings of air holes, the confinement loss is significantly reduced. On the other hand, the effective area is almost independent of the number of hole rings. We can see that the confinement loss contributes significantly to the loss of PCFs when the hole pitch $\Lambda$ is small. Fig. 10 is a general map of the effective area that can be used for designing various PCFs, such as ultralow nonlinearity PCFs and high nonlinearity PCFs with desired confinement loss properties. If, for example, 


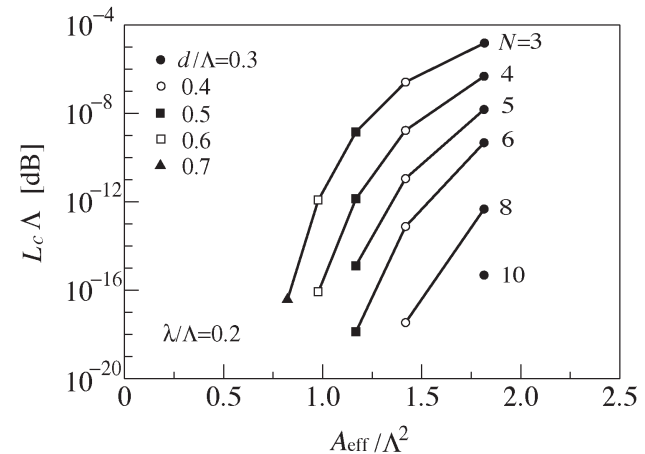

(a)

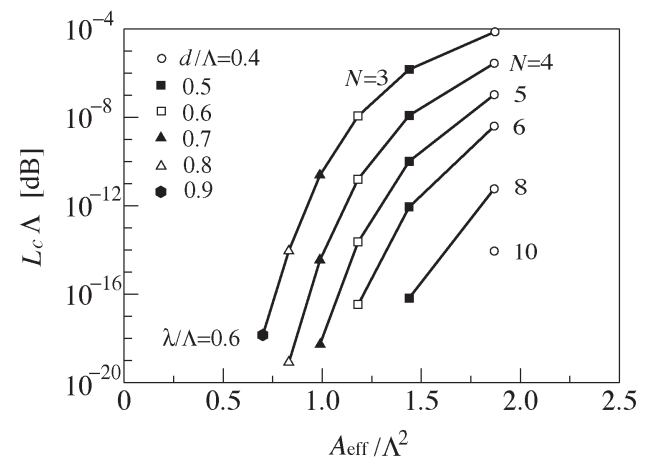

(c)

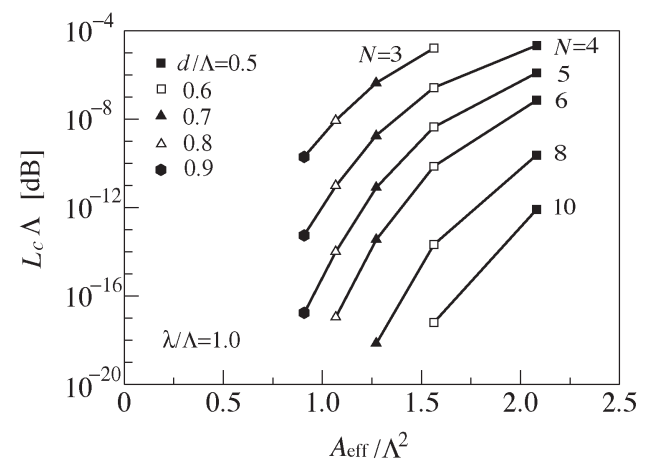

(e)

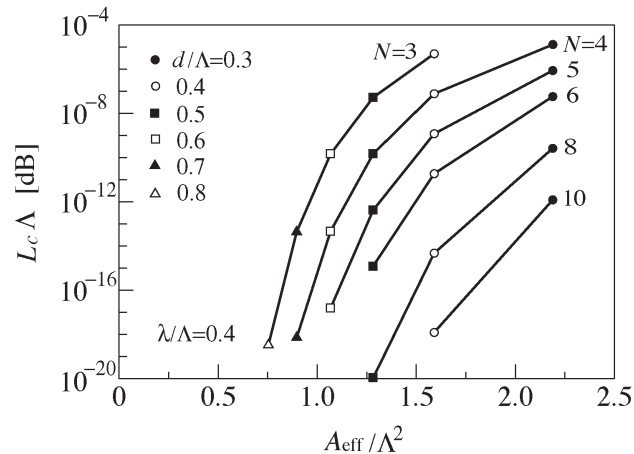

(b)

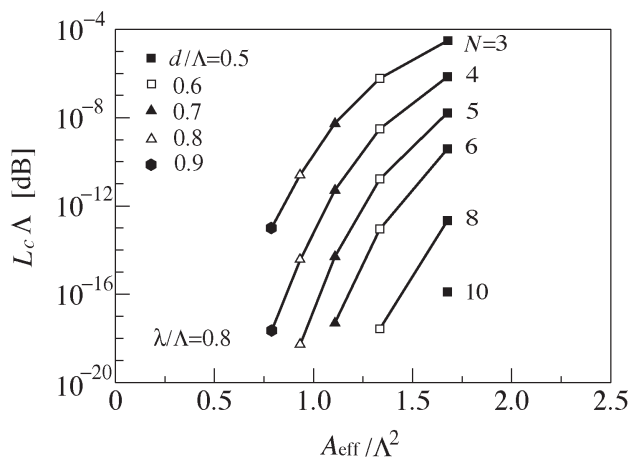

(d)

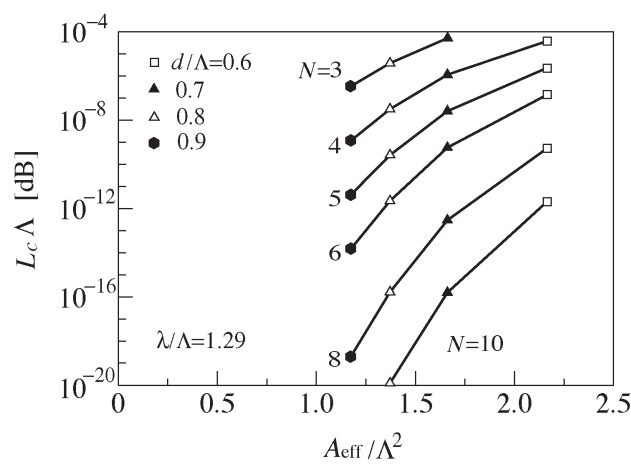

(f)

Fig. 10. Effective area and confinement loss of PCFs operating at the wavelength of (a) $\lambda / \Lambda=0.2$, (b) $\lambda / \Lambda=0.4$, (c) $\lambda / \Lambda=0.6,(d) \lambda / \Lambda=0.8$, (e) $\lambda / \Lambda=1.0$, and (f) $\lambda / \Lambda=1.29$.

the operating wavelength is given, the effective area and the confinement loss can to a large extent be tailored via the choice of hole pitch, hole size, and number of hole rings.

\section{Modal Birefringence}

One of the interesting characteristics of PCFs is their strong birefringence. Using PCFs, highly birefringent fibers can be easily realized because the index contrast is higher than conventional fibers and the fabrication process permits the formation of the required asymmetric microstructure near the fiber core. One possible use of highly birefringent PCFs is as polarization maintaining fibers (PMFs) that can stabilize the polarization state in the fiber. So far, various highly birefringent PCFs have been reported [65]-[67] as shown in Fig. 11. The configurations of Fig. 11(a) and (b) [65], [67] have different air-hole diameters along two orthogonal axes near the core region and Fig. 11(c) has two defects in the hole arrangement [66].
Fig. 12(a) shows an image generated from the scanning electron micrograph of the first reported highly birefringent PCF [64]. To estimate the birefringence of this fiber with noncircular air holes irregularly arranged around the core, it is necessary to carry out the real-model simulation [51], so the full-vector FEM is used here. The transverse electric field vector distributions of the two different polarization modes called the slow-axis and fast-axis modes are, respectively, shown in Fig. 12(b) and (c). The effective index of the slow-axis mode is slightly larger than that of the fast-axis mode. Fig. 13 shows the modal birefringence as a function of wavelength. The birefringence obtained by using the real-model simulation is $3.66 \times 10^{-3}$ at $\lambda=1.54 \mu \mathrm{m}$ and is in good agreement with the measured value of $3.7 \times 10^{-3}$ [65]. The birefringence of the order of $10^{-3}$ is one order of magnitude larger than that of conventional PMFs.

Highly birefringent PCFs have possibilities of realizing a single-polarization single-mode (SPSM) fiber [68], [69], which guides only one polarization state of the fundamental mode. 


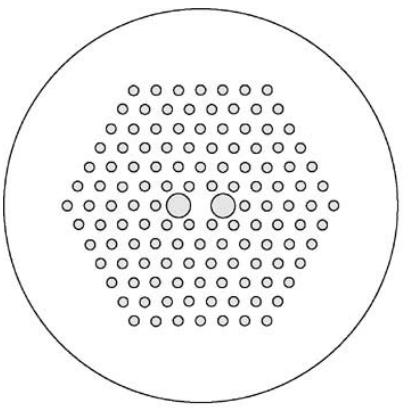

(a)

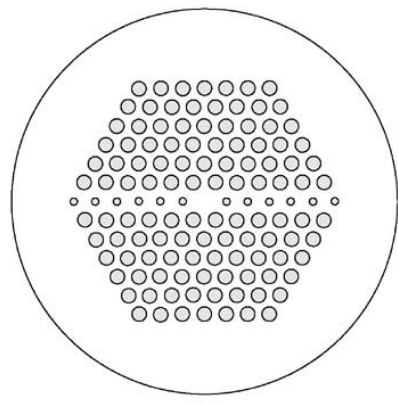

(b)

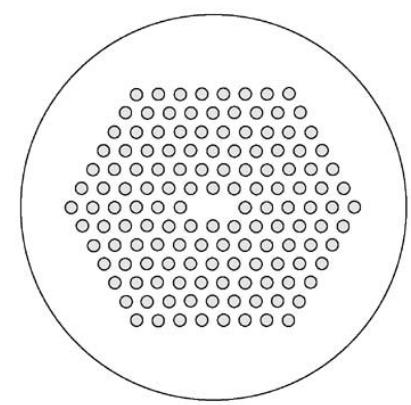

(c)

Fig. 11. Highly birefringent PCF configurations.

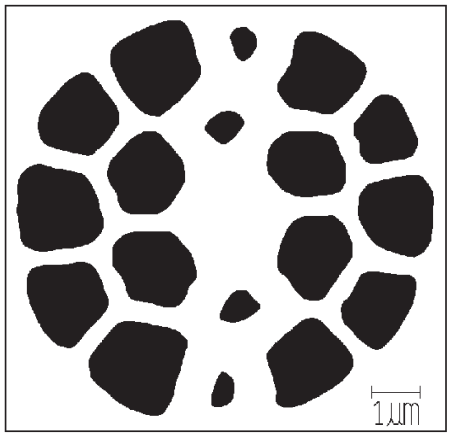

(a)

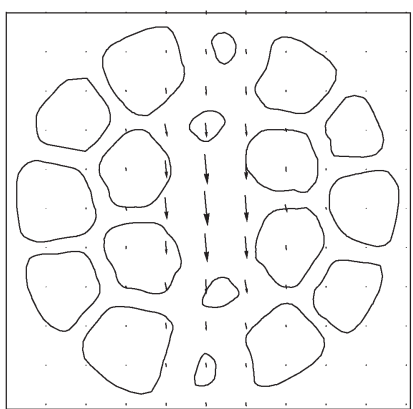

(b)

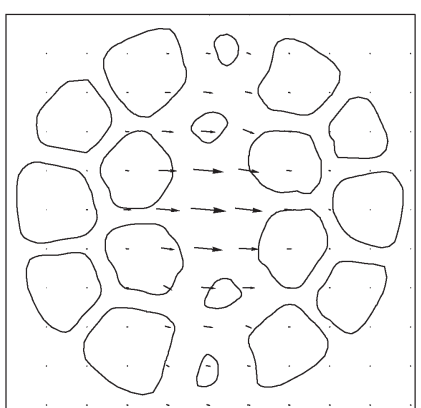

(c)
Fig. 12. (a) Highly birefringent holey fiber and the transverse electric field vector distributions of (b) slow-axis and (c) fast-axis modes.

The SPSM fiber can eliminate both polarization mode coupling and polarization mode dispersion. Fig. 14 shows an example of SPSM-PCF structure [50], [70], where $\Lambda$ is the hole pitch and $d$ and $d^{\prime}$ are the diameters of the small and large air holes, respectively. The design principle of SPSM fiber is to have the cutoff wavelengths of the fundamental modes polarized along the slow axis (slow-axis mode) and the fast axis (fastaxis mode) longer and shorter than the operating wavelength,

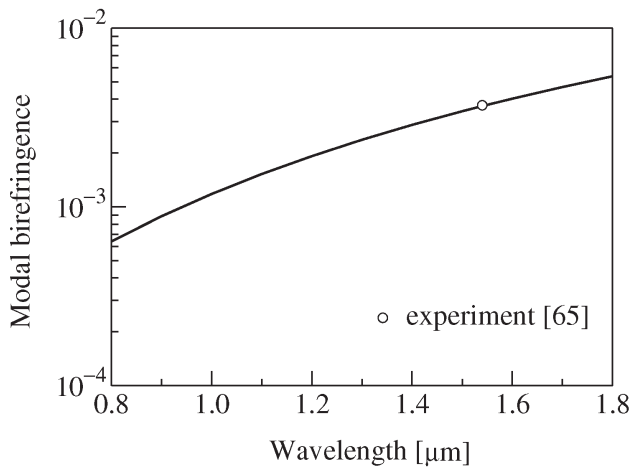

Fig. 13. Modal birefringence in the highly birefringent PCF in Fig. 12(a).

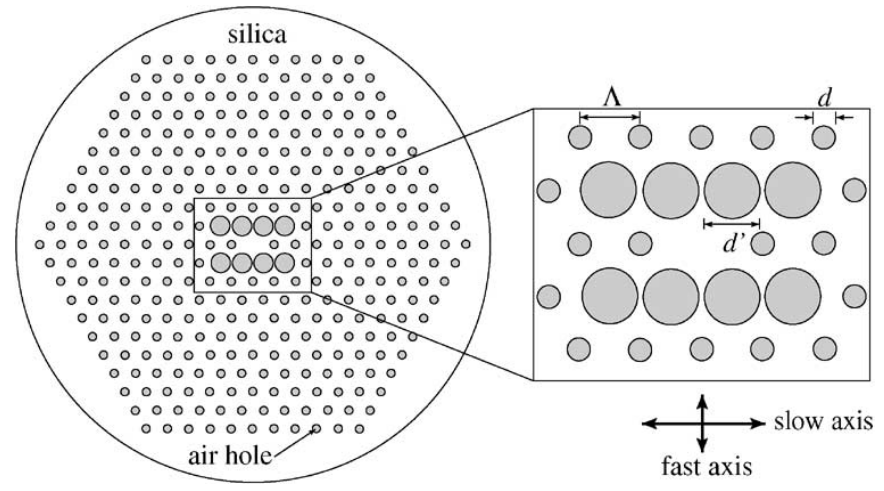

Fig. 14. Single-polarization single-mode PCF.

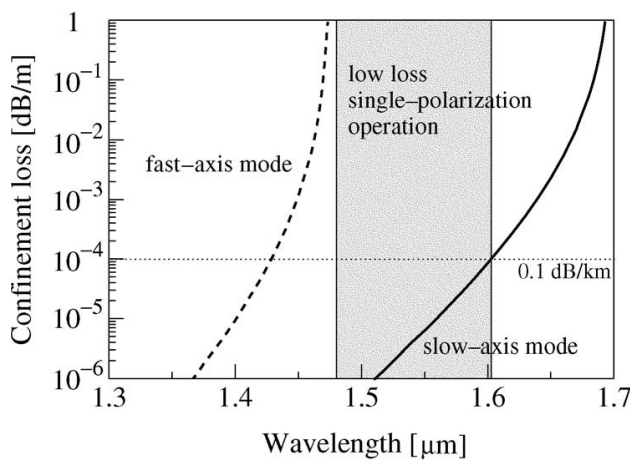

Fig. 15. Confinement loss as a function of wavelength for the singlepolarization PCF with ten rings of air holes in Fig. 14. The hole pitch $\Lambda=2.2 \mu \mathrm{m}, d / \Lambda=0.5$, and $d^{\prime} / \Lambda=0.95$.

respectively. The cutoff of either polarization occurs when its effective refractive index becomes equal to the cladding effective index of the FSM. In order to reduce the confinement loss of the slow-axis mode, eight of the central air holes are enlarged. Fig. 15 shows the wavelength dependence of the confinement loss for the single-polarization PCF with ten rings of air holes, where $\Lambda=2.2 \mu \mathrm{m}, d / \Lambda=0.5, d^{\prime}=0.95$, and the background index of silica is calculated through the Sellmeier equation. Within the wavelengths ranging from 1.48 to $1.6 \mu \mathrm{m}$, the confinement loss of the slow-axis mode is less than $0.1 \mathrm{~dB} / \mathrm{km}$ and the fast-axis mode is unguided. From these results, a low-loss SPSM-PCF can be realized with the highly birefringent PCF in Fig. 14. The SPSM-PCF is expected to be used in various optical devices as the pigtails of polarizationsensitive optical components. 
Theoretically, in the PCF structure with sixfold symmetry, the two linearly polarized fundamental modes are degenerated. Small imperfections in the PCF structure that are produced during the fabrication process, however, create asymmetries that break the degeneracy and lead to birefringence. Small irregularity, such as the variation of the hole size or the position, results in the perturbed PCF from the sixfold rotational symmetry structure. Since PCFs with the small hole pitch and the large hole diameter cause large perturbation from the perfect structure [71], [72], the PCFs with the large hole pitch and the small hole diameter have the advantage for suppressing the birefringence induced by the structural irregularity.

\section{Chromatic Dispersion TAILORING}

PCFs possess the attractive property of great controllability in chromatic dispersion. The chromatic dispersion profile can be easily controlled by varying the hole diameter and the hole pitch. Controllability of chromatic dispersion in PCFs is a very important problem for practical applications to optical communication systems, dispersion compensation, and nonlinear optics. So far, various PCFs with remarkable dispersion properties have been investigated both experimentally and numerically.

Fig. 16 shows the chromatic dispersion property of PCF with perfect hexagonal symmetry shown in Fig. 1 as a function of wavelength for $d / \Lambda$ ranging from 0.2 to 0.9 in steps of 0.1 , where the material dispersion given by a Sellmeier formula is included directly in the vector FEM calculation. The chromatic dispersion $D$ of a PCF is easily calculated from the effective index of the fundamental mode $n_{\text {eff }}$ versus the wavelength using

$$
D=-\frac{\lambda}{c} \frac{d^{2} n_{\mathrm{eff}}}{d \lambda^{2}}
$$

where $c$ is the velocity of light in a vacuum. When the hole diameter to pitch ratio is very small and the hole pitch is large, the dispersion curve is close to the material dispersion of pure silica. As the air-hole diameter is increased, the influence of waveguide dispersion becomes stronger. We can see that it is possible to shift the zero dispersion wavelength to visible to near-infrared (IR) regions by appropriately changing the geometrical parameters such as hole pitch and hole diameter [73], [74] and that a PCF with a very small hole pitch and a large air-hole diameter has large normal dispersion in the $1.55-\mu \mathrm{m}$ wavelength range [48]. In addition, Fig. 16(c) points out that a PCF with $\Lambda \approx 2.5 \mu \mathrm{m}$ and $d / \Lambda \approx 0.25$ exhibits nearly zero dispersion-flattened behavior [75], [76].

The cladding structure of typical PCFs is usually formed by air holes with the same diameter arrayed in a regular triangular lattice shown in Fig. 1. However, using a PCF with all of the same air-hole diameter in the cladding region, it is difficult to control both the dispersion and the dispersion slope in a wide wavelength range. In index-guiding PCFs, since the periodicity in the cladding region is not essential to confine the guiding light into the high-index core region, it has been reported that various effective refractive index profiles can be obtained

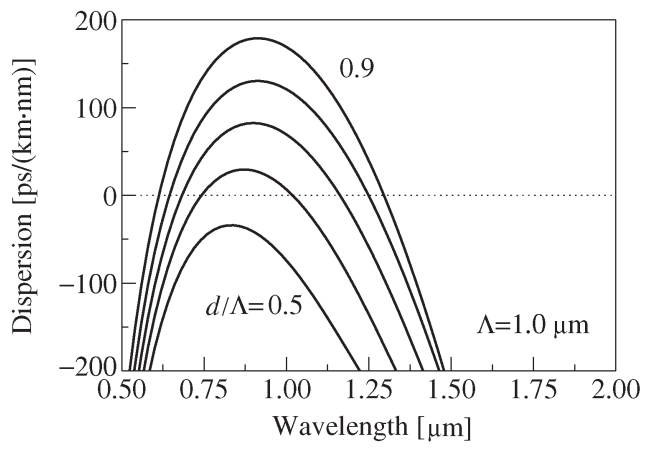

(a)

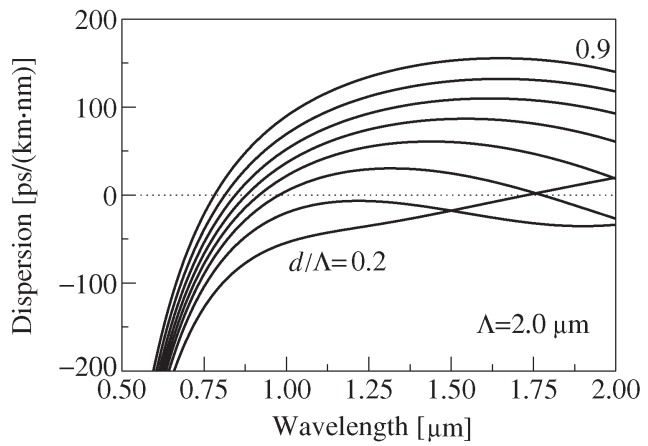

(b)

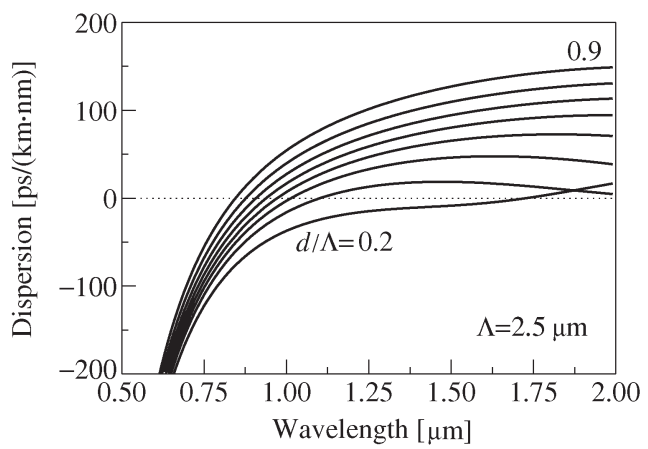

(c)

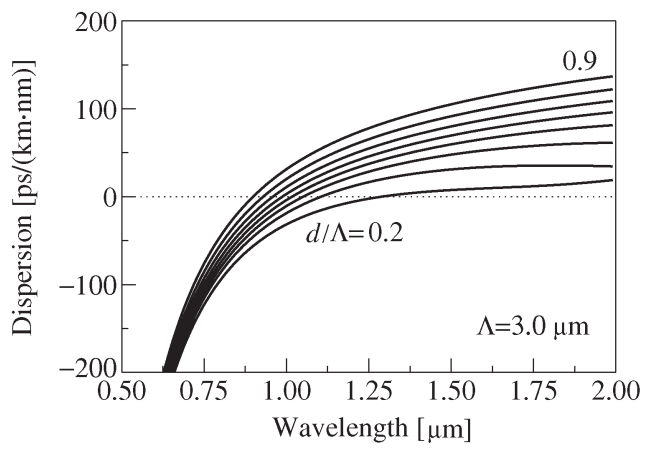

(d)

Fig. 16. Chromatic dispersion properties of PCFs for (a) $\Lambda=1.0 \mu \mathrm{m}$, (b) $\Lambda=2.0 \mu \mathrm{m}$, (c) $\Lambda=2.5 \mu \mathrm{m}$, and (d) $\Lambda=3.0 \mu \mathrm{m}$.

by varying the hole diameter of each air-hole ring along the radius [43].

Fig. 17 shows two examples of PCFs and their effective refractive index profiles, where $d_{i}(i=1$ to $n)$ is the hole diameter of the $i$ th air-hole ring and the air-hole diameters are $d_{1}>d_{2}=\cdots=d_{n}$ in Fig. 17(a) and $d_{2}<d_{1}=d_{3}=\cdots=$ $d_{n}$ in Fig. 17(b). The effective refractive index in the cladding 


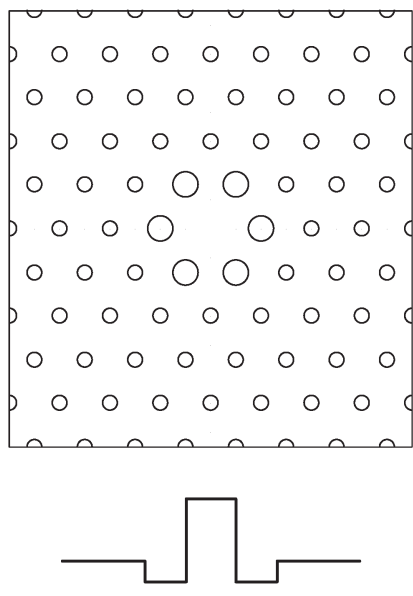

(a)
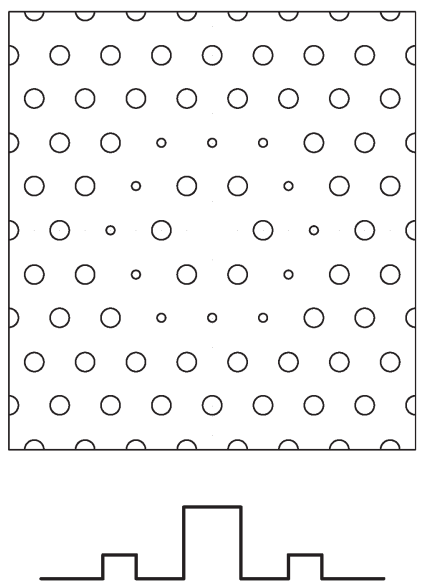

(b)
Fig. 17. Examples of PCFs and their effective refractive index profiles. The air-hole diameters are (a) $d_{1}>d_{2}=\cdots=d_{n}$ and (b) $d_{2}<d_{1}=d_{3}=$ $\cdots=d_{n}$.

region increases with decreasing air-hole diameter, and the effective refractive index in the cladding region decreases with increasing air-hole diameter. By optimizing the air-hole diameters $d_{i}$ and the hole-to-hole spacing, both the dispersion and the dispersion slope can be controlled in a wide wavelength range.

Fig. 18 shows an example of nearly zero dispersion-flattened PCF with ten air-hole rings designed by using this design principle, where $\Lambda=1.6 \mu \mathrm{m}, d_{1}=0.47 \mu \mathrm{m}, d_{2}=0.71 \mu \mathrm{m}$, $d_{3}=0.74 \mu \mathrm{m}, d_{4}=0.62 \mu \mathrm{m}, d_{5}=\cdots=d_{10}=0.65 \mu \mathrm{m}$. It is possible to decrease the effective refractive index in the cladding region along the radius by increasing the air-hole diameters along the radius. It is also possible to realize flattened dispersion by using the effective refractive index profile in Fig. 18(b) with small hole pitch.

Fig. 19 shows the chromatic dispersion and effective area as a function of wavelength for the PCF in Fig. 18(a). The wavelength range for which the PCF dispersion remains between 0.1 and $0.3 \mathrm{ps} /(\mathrm{km} \cdot \mathrm{nm})$ is from 1.41 to $1.68 \mu \mathrm{m}$. The confinement loss of the fundamental mode is less than $0.1 \mathrm{~dB} / \mathrm{km}$ in the wavelength range shorter than $1.7 \mu \mathrm{m}$. In short wavelength range, this PCF supports the second order mode; however, the confinement loss of the second-order mode is larger than $1000 \mathrm{~dB} / \mathrm{m}$ in the wavelength range over $1.0 \mu \mathrm{m}$ and the effective refractive indices of the second-order mode are quite different from those of the fundamental mode, so this PCF effectively operates as an SMF in the telecommunication window. The PCF with nearly zero flattened-dispersion and small effective area is attracting interest because it can offer high nonlinearity over a wide wavelength range [77], [78].

\section{CONCLUSION}

Analysis methods for numerical modeling of photonic crystal fibers (PCFs) were reviewed in detail. Through the full-vector finite-element method (FEM), the fundamental characteristics of PCFs such as normalized frequency, cutoff wavelength, confinement loss, modal birefringence, and chromatic dispersion have been numerically investigated. The numerical method used here can be applied to designing not only index-guiding

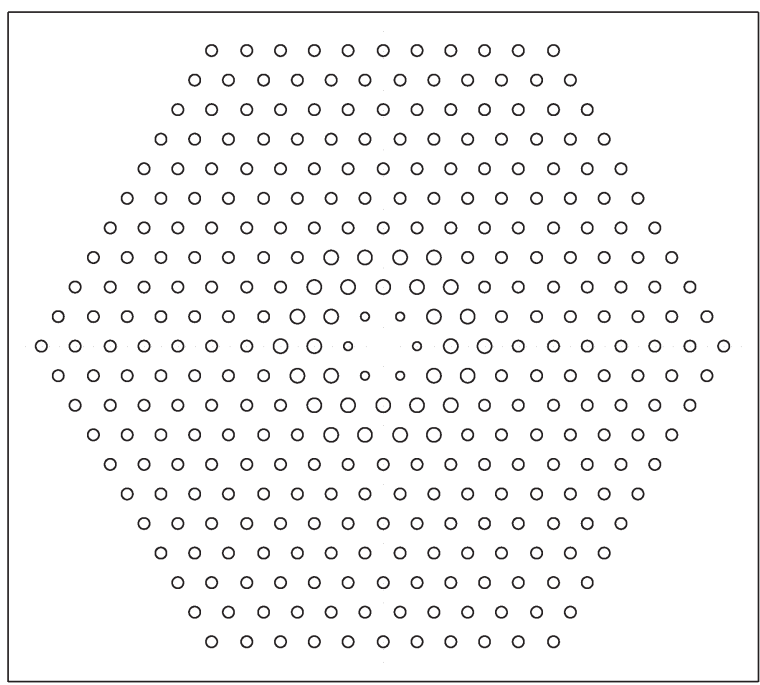

(a)

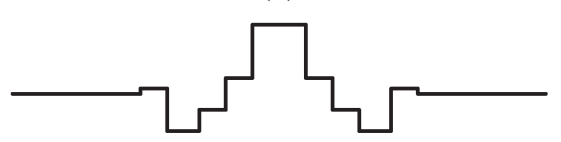

(b)

Fig. 18. (a) Nearly zero dispersion-flattened PCF with ten air-hole rings and (b) its effective refractive index profile. The hole-to-hole spacing is $\Lambda=$ $1.6 \mu \mathrm{m}$ and the air-hole diameters are $d_{1}=0.47 \mu \mathrm{m}, d_{2}=0.71 \mu \mathrm{m}, d_{3}=$ $0.74 \mu \mathrm{m}, d_{4}=0.62 \mu \mathrm{m}, d_{5}=\cdots=d_{10}=0.65 \mu \mathrm{m}$.

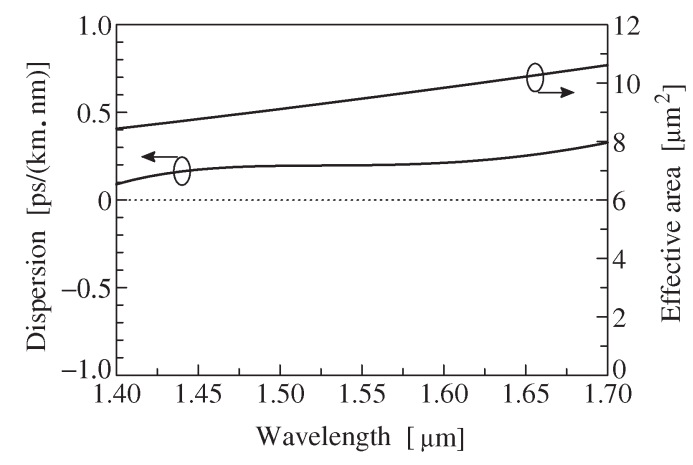

Fig. 19. Chromatic dispersion curve and effective area as a function of wavelength for dispersion-flattened PCF with ten air-hole rings in Fig. 18(a).

PCFs but also photonic band-gap fibers (PBGFs). Numerical modeling techniques have great potential for designing profitable PCFs.

\section{REFERENCES}

[1] J. Broeng, D. Mogilevstev, S. E. Barkou, and A. Bjarklev, "Photonic crystal fibers: A new class of optical waveguides," Opt. Fiber Technol., vol. 5, no. 3, pp. 305-330, Jul. 1999.

[2] T. A. Birks, J. C. Knight, B. J. Mangan, and P. S. J. Russell, "Photonic crystal fibers: An endless variety," IEICE Trans. Electron., vol. E84-C, no. 5, pp. 585-592, May 2001.

[3] J. C. Knight, "Photonic crystal fibers," Nature, vol. 424, no. 6950, pp. 847-851, Aug. 2003.

[4] J. C. Knight, T. A. Birks, P. S. J. Russell, and D. M. Atkin, "All-silica single-mode optical fiber with photonic crystal cladding," Opt. Lett., vol. 21, no. 19, pp. 1547-1549, Oct. 1996.

[5] T. A. Birks, J. C. Knight, and P. St. J. Russell, "Endlessy single-mode photonic crystal fiber,” Opt. Lett., vol. 22, no. 13, pp. 961-963, Jul. 1997.

[6] J. C. Knight, J. Broeng, T. A. Birks, and P. S. J. Russell, "Photonic band gap guidance in optical fiber," Science, vol. 282, no. 5393, pp. 1476-1478, Nov. 1998. 
[7] R. F. Cregan, B. J. Mangan, J. C. Knight, T. A. Birks, P. S. J. Russell, P. J. Roberts, and D. C. Allan, "Single-mode photonic band gap guidance of light in air," Science, vol. 285, no. 5433, pp. 1537-1539, Sep. 1999.

[8] J. C. Knight, T. A. Birks, P. S. J. Russell, and J. P. de Sandro, "Properties of photonic crystal fiber and the effective index model," J. Opt. Soc. Amer. A, vol. 15, no. 3, pp. 748-752, Mar. 1998.

[9] A. Ferrando, E. Silvester, J. J. Miret, P. Andrés, and M. V. Andrés, "Fullvector analysis of a realistic photonic crystal fiber," Opt. Lett., vol. 24, no. 5, pp. 276-278, Mar. 1999.

[10] A. Ferrando, E. Silvestre, J. J. Miret, and P. Andrés, "Vector description of higher-order modes in photonic crystal fibers," J. Opt. Soc. Amer. A, vol. 17, no. 7, pp. 1333-1340, Jul. 2000.

[11] S. E. Barkou, J. Broeng, and A. Bjarklev, "Silica-air photonic crystal fiber design that permits waveguiding by a true photonic bandgap effect," Opt. Lett., vol. 24, no. 1, pp. 46-48, Jan. 1999.

[12] J. Broeng, S. E. Barkou, T. Søndergaard, and A. Bjarklev, "Analysis of air-guiding photonic bandgap fibers," Opt. Lett., vol. 25, no. 2, pp. 96-98, Jan. 2000.

[13] D. Mogilevtsev, T. A. Birks, and P. S. J. Russell, "Group-velocity dispersion in photonic crystal fibers," Opt. Lett., vol. 23, no. 21, pp. 1662-1664, Nov. 1998.

[14] T. M. Monro, D. J. Richardson, N. G. R. Broderick, and P. J. Bennett, "Holey optical fibers: An efficient modal model," J. Lightw. Technol., vol. 17, no. 6, pp. 1093-1102, Jun. 1999.

[15] D. Mogilevtsev, T. A. Birks, and P. S. J. Russell, "Localized function method for modeling defect modes in 2-D photonic crystals," J. Lightw. Technol., vol. 17, no. 11, pp. 2078-2081, Nov. 1999.

[16] T. M. Monro, D. J. Richardson, N. G. R. Broderick, and P. J. Bennett, "Modeling large air fraction holey optical fibers," J. Lightw. Technol., vol. 18, no. 1, pp. 50-56, Jan. 2000.

[17] M. J. Steel, T. P. White, C. M. de Sterke, R. C. McPhedran, and L. C. Botten, "Symmetry and degeneracy in microstructured optical fibers," Opt. Lett., vol. 26, no. 8, pp. 488-490, Apr. 2001.

[18] T. P. White, R. C. McPhedran, C. M. de Sterke, L. C. Botten, and M. J. Steel, "Confinement losses in microstructured optical fibers," Opt. Lett., vol. 26, no. 21, pp. 1660-1662, Nov. 2001.

[19] T. P. White, R. C. McPhedran, L. C. Botten, G. H. Smith, and C. M. de Sterke. (2001, Dec.). Calculations of air-guided modes in photonic crystal fibers using the multipole method. Opt. Express [Online]. 9(13), pp. 721-732. Available: http://www.opticsexpress.org/

[20] T. P. White, B. T. Kuhlmey, R. C. McPhedran, D. Maystre, G. Renversez, C. M. de Sterke, and L. C. Botten, "Multipole method for microstructured optical fibers. I. Formulation," J. Opt. Soc. Amer. B, vol. 19, no. 10, pp. 2322-2330, Oct. 2002.

[21] B. T. Kuhlmey, T. P. White, G. Renversez, D. Maystre, L. C. Botten, C. M. de Sterke, and R. C. McPhedran, "Multipole method for microstructured optical fibers. II. Implementation and results," J. Opt. Soc. Amer. B, vol. 19 , no. 10 , pp. 2331-2340, Oct. 2002.

[22] C. E. Kerbage, B. J. Eggleton, P. S. Westbrook, and R. S. Windeler. (2000, Jul.). Experimental and scalar beam propagation analysis of an air-silica microstructure fiber. Opt. Express [Online]. 7(3), pp. 113-122. Available: http://www.opticsexpress.org/

[23] B. J. Eggleton, P. S. Westbrook, C. A. White, C. Kerbage, R. S. Windeler, and G. L. Burdge, "Cladding-mode resonances in air-silica microstructure optical fibers," J. Lightw. Technol., vol. 18, no. 8, pp. 1084-1100, Aug. 2000.

[24] F. Fogli, L. Saccomandi, and P. Bassi. (2002, Jan.). Full vectorial BPM modeling of index-guiding photonic crystal fibers and couplers. Opt. Express [Online]. 10(1), pp. 54-59. Available: http://www.opticsexpress.org/

[25] Z. Zhu and T. G. Brown. (2002, Aug.). Full-vectorial finite-difference analysis of microstructured optical fibers. Opt. Express [Online]. 10(17), pp. 853-864. Available: http://www.opticsexpress.org/

[26] G. E. Town and J. T. Lizer, "Tapered holey fibers for spot-size and numerical-aperture conversion," Opt. Lett., vol. 26, no. 14, pp. 10421044, Jul. 2001

[27] J. T. Lizier and G. E. Town, "Splice losses in holey optical fibers," IEEE Photon. Technol. Lett., vol. 13, no. 8, pp. 794-796, Aug. 2001.

[28] M. Qiu, "Analysis of guided modes in photonic crystal fibers using the finite-difference time-domain method," Microw. Opt. Technol. Lett., vol. 30, no. 5, pp. 327-330, Sep. 2001.

[29] N. Guan, S. Habu, K. Takenaga, K. Himeno, and A. Wada, "Boundary element method for analysis of holey optical fibers," J. Lightw. Technol., vol. 21, no. 8, pp. 1787-1792, Aug. 2003.

[30] T.-L. Wu and C.-H. Chao, "Photonic crystal fiber analysis through the vector boundary-element method: Effect of elliptical air hole," IEEE Photon. Technol. Lett., vol. 16, no. 1, pp. 126-128, Jan. 2004.

[31] F. Brechet, J. Marcou, D. Pagnoux, and P. Roy, "Complete analysis of the characteristics of propagation into photonic crystal fibers, by the finite element method," Opt. Fiber Technol., vol. 6, no. 2, pp. 181-191, Apr. 2000.

[32] M. Koshiba and K. Saitoh, "Numerical verification of degeneracy in hexagonal photonic crystal fibers," IEEE Photon. Technol. Lett., vol. 13, no. 12, pp. 1313-1315, Dec. 2001.

[33] S. Guenneau, A. Nicholet, F. Zolla, and S. Lasquellec, "Modeling of photonic crystal optical fibers with finite elements," IEEE Trans. Magn., vol. 38, no. 2, pp. 1261-1264, Mar. 2002.

[34] M. Koshiba, "Full-vector analysis of photonic crystal fibers using the finite element method," IEICE Trans. Electron., vol. 85-C, no. 4, pp. 881888, Apr. 2002

[35] K. Saitoh and M. Koshiba, "Full-vectorial imaginary-distance beam propagation method based on a finite element scheme: Application to photonic crystal fibers," IEEE J. Quantum Electron., vol. 38, no. 7, pp. 927-933, Jul. 2002.

[36] A. Cucinotta, S. Selleri, L. Vincetti, and M. Zoboli, "Perturbation analysis of dispersion properties in photonic crystal fibers through the finite element method," J. Lightw. Technol., vol. 20, no. 8, pp. 1433-1442, Aug. 2002.

[37] K. Saitoh and M. Koshiba, "Photonic bandgap fibers with high birefringence," IEEE Photon. Technol. Lett., vol. 14, no. 9, pp. 1291-1293, Sep. 2002.

[38] A. Cucinotta, S. Selleri, L. Vincetti, and M. Zoboli, "Holey fiber analysis through the finite-element method," IEEE Photon. Technol. Lett., vol. 14 no. 11 , pp. 1530-1532, Nov. 2002.

[39] D. Ferrarini, L. Vincetti, M. Zoboli, A. Cucinotta, and S. Selleri. (2002, Nov.). Leakage properties of photonic crystal fibers. Opt. Express [Online]. 10(23), pp. 1314-1319. Available: http://www.opticsexpress.org/

[40] K. Saitoh and M. Koshiba, "Confinement losses in air-guiding photonic bandgap fibers," IEEE Photon. Technol. Lett., vol. 15, no. 2, pp. 236-238, Feb. 2003.

[41] A. Perilloux, T. Chartier, A. Hinder, L. Berthelot, G. Melin, S. Lempereur, D. Pagnoux, and P. Roy, "Theoretical and experimental study of the birefringence of a photonic crystal fiber," J. Lightw. Technol., vol. 21, no. 2, pp. 536-539, Feb. 2003.

[42] A. Cucinotta, F. Poli, S. Selleri, L. Vincetti, and M. Zoboli, "Amplification properties of $\mathrm{Er}^{3+}$-doped photonic crystal fibers," J. Lightw. Technol., vol. 21, no. 3, pp. 782-788, Mar. 2003.

[43] K. Saitoh, M. Koshiba, T. Hasegawa, and E. Sasaoka. (2003, Apr.) Chromatic dispersion control in photonic crystal fibers: Application to ultra-flattened dispersion. Opt. Express [Online]. 11(8), pp. 843-852. Available: http://www.opticsexpress.org/

[44] M. Koshiba and K. Saitoh, "Polarization-dependent confinement losses in actual holey fibers," IEEE Photon. Technol. Lett., vol. 15, no. 5, pp. 691693, May 2003

[45] T. Fujisawa and M. Koshiba. (2003, Jun.). Finite element characterization of chromatic dispersion in nonlinear holey fibers. Opt. Express [Online]. 11(13), pp. 1481-1489. Available: http://www.opticsexpress.org/

[46] M. Koshiba and K. Saitoh. (2003, Jul.). Structural dependence of effective area and mode field diameter for holey fibers. Opt. Express [Online]. 11(15), pp. 1746-1756. Available: http://www.opticsexpress.org/

[47] J. Jin, W. Jin, and M. S. Demokan, "Properties of a highly birefringent photonic crystal fiber," IEEE Photon. Technol. Lett., vol. 15, no. 10, pp. 1375-1377, Oct. 2003.

[48] F. Poli, A. Cucinotta, M. Fuochi, S. Selleri, and L. Vincetti, "Characterization of microstructured optical fibers for wideband dispersion compensation," J. Opt. Soc. Amer. A, vol. 20, no. 10, pp. 1958-1962, Oct. 2003.

[49] M. Fuochi, F. Poli, S. Selleri, A. Cucinotta, and L. Vincetti, "Study of Raman amplification properties in triangular photonic crystal fibers," J. Lightw. Technol., vol. 21, no. 10, pp. 2247-2254, Oct. 2003.

[50] K. Saitoh and M. Koshiba, "Single-polarization single-mode photonic crystal fibers," IEEE Photon. Technol. Lett., vol. 15, no. 10, pp. 1384 1386, Oct. 2003.

[51] M. Koshiba and K. Saitoh, "Finite-element analysis of birefringence and dispersion properties in actual and idealized holey-fiber structures," Appl. Opt., vol. 42, no. 31, pp. 6267-6275, Nov. 2003.

[52] K. Saitoh and M. Koshiba. (2003, Nov.). Leakage loss and group velocity dispersion in air-core photonic bandgap fibers. Opt. Express [Online]. 11(23), pp. 3100-3109. Available: http://www.opticsexpress.org/

[53] K. Saitoh, Y. Sato, and M. Koshiba. (2003, Dec.). Coupling characteristics of dual-core photonic crystal fiber couplers. Opt. Express [Online]. 11(24), pp. 3188-3195. Available: http://www.opticsexpress.org/

[54] K. Saitoh, N. A. Mortensen, and M. Koshiba. (2004, Feb.). Air-core photonic band-gap fibers: The impact of surface modes. Opt. Express [Online]. 12(3), pp. 394-400. Available: http://www.opticsexpress.org/ 
[55] M. Koshiba and Y. Tsuji, "Curvilinear hybrid edge/nodal elements with triangular shape for guided-wave problems," J. Lightw. Technol., vol. 18, no. 5, pp. 737-743, May 2000.

[56] M. Midro, M. P. Singh, and C. G. Someda, "The space filling mode of holey fibers: An analytical vectorial solution," J. Lightw. Technol., vol. 18, no. 7, pp. 1031-1037, Jul. 2000

[57] M. Koshiba and K. Saitoh, "Applicability of classical optical fiber theories to holey fibers," Opt. Lett., vol. 29, no. 15, pp. 1739-1741, Aug. 2004.

[58] N. A. Mortensen, J. R. Folkenberg, M. D. Nielsen, and K. P. Hansen, "Modal cutoff and the V parameter in photonic crystal fibers," Opt. Lett., vol. 28, no. 20, pp. 1879-1881, Oct. 2003.

[59] J. R. Folkenberg, N. A. Mortensen, K. P. Hansen, T. P. Hansen, H. R. Simonsen, and C. Jakobsen, "Experimental investigation of cutoff phenomena in nonlinear photonic crystal fibers," Opt. Lett., vol. 28, no. 20, pp. 1882-1884, Oct. 2003.

[60] D. Marcuse, "Loss analysis of single-mode fiber splices," Bell Syst. Tech. J., vol. 56, no. 5, pp. 703-718, May-Jun. 1977.

[61] M. D. Nielsen, C. Jacobsen, N. A. Mortensen, J. R. Folkenberg, and H. R. Simonsen. (2004, Apr.). Low-loss photonic crystal fibers for transmission systems and their dispersion properties. Opt. Express [Online]. 12(7), pp. 1372-1376. Available: http://www.opticsexpress.org/

[62] J. Ju, W. Jin, Y. L. Hoo, and M. S. Demokan, "A simple method for estimating the splice loss of photonic-crystal fiber/single-mode fiber," Microw. Opt. Technol. Lett., vol. 42, no. 2, pp. 171-173, May 2004.

[63] K. Tajima, J. Zhou, K. Kurokawa, and K. Nakajima, "Low water peak photonic crystal fibers," presented at the Eur. Conf. Optical Communication (ECOC), Rimini, Italy, Sep. 2003, Th4.1.6.

[64] G. Agrawal, Nonlinear Fiber Optics, 2nd ed. San Diego, CA: Academic, 1995.

[65] A. Ortigosa-Blanch, J. C. Knight, W. J. Wadsworth, J. Arriaga, B. J. Mangan, T. A. Birks, and P. S. J. Russell, "Highly birefringent photonic crystal fibers," Opt. Lett., vol. 25, no. 18, pp. 1325-1327, Sep. 2000.

[66] T. P. Hansen, J. Broeng, S. E. B. Libori, E. Knuders, A. Bjarklev, J. R. Jensen, and H. Simonsen, "Highly birefringent index-guiding photonic crystal fibers," IEEE Photon. Technol. Lett., vol. 13, no. 6, pp. 588590, Jun. 2001.

[67] K. Suzuki, H. Kubota, S. Kawanishi, M. Tanaka, and M. Fujita. (2001, Dec.). Optical properties of a low-loss polarization-maintaining photonic crystal fiber. Opt. Express [Online]. 9(13), pp. 676-680. Available: http://www.opticsexpress.org/

[68] J. R. Simpson, R. H. Stolen, F. M. Sears, W. Pleibel, J. B. Macchesney, and R. E. Howard, "A single-polarization fiber," J. Lightw. Technol., vol. LT-1, no. 2, pp. 370-373, Jun. 1983.

[69] H. Kubota, S. Kawanishi, S. Koyanagi, M. Tanaka, and S. Yamaguchi, "Absolutely single polarization photonic crystal fiber," IEEE Photon. Technol. Lett., vol. 16, no. 1, pp. 182-184, Jan. 2004.

[70] N. Guan, K. Takenaga, K. Izoe, R. Suzuki, and K. Himeno, "Highly birefringent photonic crystal fiber for a wide wavelength range," presented at the Eur. Conf. Optical Communication (ECOC), Stockholm, Sweden, Sep. 2004, Mo4.3.4.

[71] I.-K. Hwang, Y.-J. Lee, and Y.-H. Lee. (2003, Nov.). Birefringence induced by irregular structure in photonic crystal fiber. Opt. Express [Online]. 11(22), pp. 2799-2806. Available: http://www.opticsexpress.org/

[72] K. L. Reichenbach, and C. Xu. (2005, Apr.). The effects of randomly occurring nonuniformities on propagation in photonic crystal fibers. Opt. Express [Online]. 13(8), pp. 2799-2807. Available: http://www.opticsexpress.org/

[73] J. C. Knight, J. Arriaga, T. A. Birks, A. Ortigosa-Blanch, W. J. Wadsworth, and P. S. J. Russell, "Anomalous dispersion in photonic crystal fiber," IEEE Photon. Technol. Lett., vol. 12, no. 7, pp. 807-809, Jul. 2000.

[74] D. Ouzounov, D. Homoelle, W. Zipfel, W. W. Webb, A. L. Gaeta, J. A. West, J. C. Fajardo, and K. W. Koch, "Dispersion measurements of microstructured fibers using femtosecond laser pulses," Opt. Commun., vol. 192, no. 3-6, pp. 219-223, Jun. 2001.

[75] A. Ferrando, E. Silvestre, J. J. Miret, and P. Andrés, "Nearly zero ultraflattened dispersion in photonic crystal fibers," Opt. Lett., vol. 25, no. 11, pp. 790-792, Jun. 2000.

[76] W. H. Reeves, J. C. Knight, P. S. J. Russell, and P. J. Roberts (2002, Jul.). Demonstration of ultra-flattened dispersion in photonic crystal fibers. Opt. Express [Online]. 10(14), pp. 609-613. Available: http://www.opticsexpress.org/
[77] T. Yamamoto, H. Kubota, S. Kawanishi, M. Tanaka, and S. Yamaguchi. (2003, Jun.). Supercontinuum generation at $1.55 \mu \mathrm{m}$ in a dispersion-flattened polarization-maintaining photonic crystal fiber. Opt. Express [Online]. 11(13), pp. 1537-1540. Available: http://www. opticsexpress.org/

[78] K. Saitoh, and M. Koshiba. (2004, May). Highly nonlinear dispersionflattened photonic crystal fibers for supercontinuum generation in a telecommunication window. Opt. Express [Online]. 12(10), pp. 2027-2032. Available: http://www.opticsexpress.org/

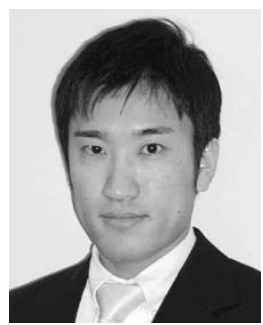

Kunimasa Saitoh (S'00-M'01) was born in Hokkaido, Japan, on July 30, 1973. He received the B.S., M.S., and Ph.D. degrees in electronic engineering from Hokkaido University, Sapporo, Japan, in 1997, 1999, and 2001, respectively.

From 1999 to 2001, he was a Research Fellow at the Japan Society for the Promotion of Science. From 2001 to 2005, he was a Research Associate at the Graduate School of Engineering, Hokkaido University. In 2005, he became an Associate Professor at the Graduate School of Information Science and Technology, Hokkaido University. He has been engaged in research on wave electronics, including lightwaves, surface acoustic waves, and computer-aided design and modeling of guided-wave devices using the finite-element method, beam propagation method, and so on.

Dr. Saitoh is a Member of the Institute of Electronics, Information and Communication Engineers (IEICE). In 1999 and 2002, he was awarded the Excellent Paper Award and the Young Scientist Award from the IEICE, respectively.

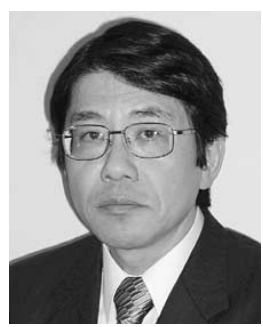

Masanori Koshiba (SM'84-F'03) was born in Sapporo, Japan, on November 23, 1948. He received the B.S., M.S., and Ph.D. degrees in electronic engineering from Hokkaido University, Sapporo, in 1971, 1973, and 1976, respectively.

In 1976, he joined the Department of Electronic Engineering, Kitami Institute of Technology, Kitami, Japan. From 1979 to 1987, he was an Associate Professor of Electronic Engineering at Hokkaido University, where, in 1987, he became a Professor. He has been engaged in research on wave electronics, including microwaves, millimeter-waves, lightwaves, surface acoustic waves (SAW), magnetostatic waves (MSW), and electron waves, and computer-aided design and modeling of guided-wave devices using the finite-element method, boundary element method, beam propagation method, and so on. He is the author and/or co-author of more than 260 research papers in English and of more than 130 research papers in Japanese both in refereed journals. He is the author of the books Optical Waveguide Analysis (New York: McGrawHill, 1992) and Optical Waveguide Theory by the Finite Element Method (Tokyo, Japan: KTK Scientific/Dordrecht, The Netherlands: Kluwer Academic, 1992), and is the co-author of the books Analysis Methods for Electromagnetic Wave Problems (Boston, MA: Artech House, 1990), Analysis Methods for Electromagnetic Wave Problems, Vol. Two (Boston, MA: Artech House, 1996), Ultrafast and Ultra-parallel Optoelectronics (Chichester, U.K.: Wiley, 1995), and Finite Element Software for Microwave Engineering (New York: Wiley, 1996).

Dr. Koshiba is a Fellow of the Institute of Electronics, Information and Communication Engineers (IEICE) and is a Member of the Institute of Electrical Engineers of Japan, the Institute of Image Information and Television Engineers of Japan, the Japan Society for Simulation Technology, and the Japan Society for Computational Methods in Engineering. In 1987, 1997, and 1999, he received the Excellent Paper Awards from the IEICE, in 1998 the Electronics Award from the IEICE-Electronics Society, and in 2004 the Achievement Award from the IEICE. From 1999 to 2000, he served as the President of the IEICE-Electronics Society, and in 2002 as the Chair of the IEEE Lasers and Electro-Optics Society (LEOS) Japan Chapter. Since 2003, he had served on the Board of Directors of the IEICE. 